\title{
As abordagens pedagogicas e o papel do professor de educação básica em processo de formação continuada no brasil: uma revisão sistemática da literatura
}

\section{The pedagogical approaches and the role of the basic education teacher's in a continued training process in brazil: a systematic review}

\author{
Simone Maneira, Maria João Gomes \\ Universidade do Minho
}

\begin{abstract}
Resumen
Importa para este artigo conhecer quais as abordagens formativas que se fazem presentes na composição das propostas de formação continuada em TIC no Brasil. Compreende-se que as mesmas exercem um diferencial importante e repercutem na ação, na reflexão e no compromisso docente com as Tecnologias Digitais. Neste Sentido, busca-se por meio de uma Revisão Sistemática da Literatura encontrar evidências capazes de nos levar a perceber as influências da abordagem pedagógica no papel do professor enquanto aprendiz de TIC. Este estudo ressalta a formação docente como o espaço de diálogo entre as demandas do Século XXI e a situacionalidade em TIC.

Palavras-chave: abordagem, formação continuada, situacionalidade docente em TIC
\end{abstract}

\begin{abstract}
It is important for this article to know which training approaches are present in the composition of the proposals for continuing training in ICT in Brazil. It is understood that they exert an important differential and have repercussions on the action, the reflection and the teaching commitment with the Digital Technologies. In this sense, it is sought through a Systematic Review to find evidence capable of leading us to perceive the influences of the pedagogical approach in the role of the teacher as an apprentice of ICT. This study seeks to emphasize teacher education as the space for dialogue between the demands of the 21st Century and the situationality in ICT.

Keywords: Approach, continuing education, ICT teaching situationality
\end{abstract}

\section{Situacionalidade docente em TIC}

De acordo com um estudo realizado pelo movimento da sociedade brasileira Todos pela Educação (TPE), e intitulado de Formação de Professores no Brasil Diagnóstico, agenda de políticas e estratégias para a mudança (2016), chegou-se a informação de que a "precariedade da formação inicial é amplificada pelo baixo capital cultural e social da grande maioria dos estudantes de magistério. Isso faz com que a garantia de uma boa formação continuada ganhe ainda mais relevância" (p. 16). Dado a citação, percebe-se as apostas que se fazem aos processos de capacitação docente pós- universidade, e que contemplam o professor dentro de sua profissionalização. Mas a questão da "precariedade" da formação nos preocupa, levando-nos a questionar: qual é a situacionalidade docente em Tecnologias da Informação e da Comunicação (TIC) dentro deste cenário, e quais seus condicionantes.

A ideia da expressão "situacionalidade docente em TIC" nasce na reflexão sobre o professor e sua relação com as tecnologias digitais. Visualizamos neste horizonte o enfrentamento do professor às demandas postuladas pelo rápido desenvolvimento da tecnologia e de sua extensão à educação. Nesta arena, entendemos o professor como um sujeito que não está apenas no mundo em que a tecnologia se desenvolve, mas que está com ele, ou seja, tem uma relação com este mundo. A reflexão apresentada vem influenciada por Freire (1979) por meio de sua obra Educação e Mudança. Foi ela quem nos levou a pensar e discutir o professor diante de três verbos que evidenciam esta relação com o mundo das tecnologias digitais: ação, reflexão e compromisso. É neste tríduo freiriano que buscamos fundamentar a relação do professor e suas respostas diante das demandas tecnológicas. Por isso da expressão situacionalidade docente em TIC, que por ora reconhecemos como um conjunto de fatores que impactuam na ação, na reflexão e no compromisso dos professores com as Tecnologias da Informação e Comunicação.

Deste modo, o pensar a situacionalidade docente em TIC remete à busca da compreensão do professor dentro de sua realidade laboral, ou seja, dentro do conjunto de fatores que repercutem em sua práxis no que tange o trabalho com as TIC. Por este motivo associamos a ideia de situacionalidade docente em TIC com a instância da formação, sobretudo aquela de cunho continuado, percebendo nela um importante papel no desenvolvimento das TIC dentro do tríduo ação, reflexão e compromisso docente. De fato, não há como desvendar a conexão docente com as TIC, sem pensar a questão dos processos de formação docente e seus condicionantes. É nesta base que este artigo se ampara, buscando saber 
quais as abordagens formativas que se fazem presentes na composição das propostas de formação em TIC, especificamente em relação as propostas de formação continuada dos professores brasileiros de Educação Básica e pública, identificando também que influências estas abordagens trazem ao papel do professor enquanto aprendiz de TIC. Para o desenvolvimento deste propósito, faremos uso da Revisão Sistemática da Literatura que contemplará os estudos primários que tratam de formação continuada de forma empírica, dando atenção aos que também mencionam o Technological Pedagogical Content Knowledge (TPACK) em seu desenvolvimento. Imaginamos que este trabalho poderá repercutir como um contributo de compreensão e possível intervenção nos processos de formação continuada em sua relação com a situacionalidade docente em TIC.

\section{Abordagem formativa e o papel do professor enquanto aprendiz de TIC}

É preciso clarificar que quando estamos falando em abordagem pedagógica, queremos nos referir às formas operacionais que os processos de formação continuada apresentam para disseminar o conhecimento TIC aos docentes em contexto de formação. Em Valente (1998) podemos encontrar uma preocupação neste sentido, quando o mesmo coloca que os processos de formação devem prover condições ao professor para que possa construir o conhecimento em tecnologia e entender o como este conhecimento pode ser integrado em sua prática. Nesse sentido, pensar a ideia da abordagem pedagógica, a nosso ver, vai de encontro ao pensar o papel do professor enquanto aprendiz de TIC, sendo este papel o diferencial para que o mesmo possa, e aqui terminamos nosso raciocínio com Valente (1989, p.2) "recontextualizar o aprendizado e a experiência vivida durante a sua formação para a sua realidade de sala de aula, compatibilizando as necessidades de seus alunos e os objetivos pedagógicos que se dispõe a atingir".

\section{Metodologia}

A RSL efetivada neste trabalho contou com o referencial de Boland, Cherry \& Dickson (2013), apresentando consideração às etapas propostas no seu desenvolvimento. Neste trabalho, e tendo em vista as leituras e estudos já realizados ao longo do desenvolvimento de nossa jornada doutoral, chegamos às palavras-chaves para a nossa busca nas bases de dados. Assim, compreendemos que "TPACK", ou "TPCK", "formação continuada", "educação básica”, e "educação brasileira", como os descritores de base para a elaboração de uma string de busca mais detalhada. Deste modo tratamos os mesmos de acordo com às características de cada base de dados, potenciando-os na língua inglesa: "TPACK", "TPCK", "continuous training", "continuous teacher training", "professional development", "professional teacher development", "teacher development", e "brasilian education". As bases de dados consultadas foram: b-ON (Biblioteca do conhecimento online); Emerald; ERIC (Education Resources Information Centre); IEEE (Institute of Electrical and Electronics Engineers); Portal de Periódicos da Capes
(Google Acadêmico); Oasisbr (Portal Brasileiro de Publicações Científicas de Acesso Aberto); e Redalyc (Rede de Revistas Científicas da América Latina e Caribe, Espanha e Portugal).

Em relação aos critérios de inclusão, consideramos a nossa questão principal que visou buscar quais as abordagens formativas que se fazem presentes na composição das propostas de formação continuada em TIC dos professores brasileiros de Educação Básica, dando atenção à identificação das influências que as abordagens estruturadoras destas propostas trazem ao papel do professor enquanto aprendiz de TIC. Portanto, consideramos como critérios de inclusão cinco pontos pertinentes: estudos empíricos; que retratem propostas de formação continuada fundamentadas no TPACK; que estejam no contexto brasileiro de Educação Básica; entre os anos de 2006 a 2016; e na língua inglesa, portuguesa ou espanhola. Justifica-se a escolha dos processos de formação que retratem o TPACK porque percebemos que o mesmo pode ser considerado um elemento científico de intervenção estruturante dos processos de formação continuada (Maneira, Martins \& Gomes, 2017). Além do mais o referencial teórico do TPACK representa o como os professores podem fazer a intersecção entre os conhecimento do conteúdo, da pedagogia e da tecnologia (Mishra \& Koehler, 2006).

Por meio da operacionalização dos descritores na ferramenta de busca das bases de dados, chegamos ao número de 126 estudos. O trabalho de leitura de seus títulos e resumos reduziu este número para 46 estudos relevantes para a solução da nossa questão principal, no entanto, após o filtro estabelecido pelos critérios de inclusão e exclusão, somente 8 estudos foram considerados potencialmente relevantes para nossa investigação. No entanto, é preciso justificar a exclusão de muitos dos artigos, colocando que 6 foram excluídos por abordarem a formação inicial; 4 por serem duplicados; 5 devido ao acesso restrito; e 23 artigos porque tratavam de outros assuntos pertinentes ao campo da educação. Os estudos incluídos foram submetidos a um processo de avaliação que considerou a adaptação do instrumento da Critical Appraisal Skills Programme (CASP), sendo todos eles classificados como estudos de qualidade, com rigor, credibilidade e relevantes no que tange aos aspectos mais evidentes.

Para a realização da etapa de extração de dados da RSL, elencamos alguns critérios de análise que também consideraram nossa problemática junto ao objetivo. Deste modo construímos uma planilha no Excel elencando todos eles para uma extração de dados dirigida. Entre estes critérios estão: a problemática dos estudos; seus objetivos; a abordagem formativa; as características da formação em TPACK; a população; e o papel do professor participante na formação. Entendemos que, embora tenhamos elegido estes seis critérios, cada estudo revelará o seu contexto de ação, e as influências deste nesta ação. Por esta razão, decidimos pela RSL uma vez que este método de revisão se concentra num trabalho crítico-reflexivo. 


\section{Resultados}

Com a análise dos 8 estudos incluídos, pudemos perceber que o Construcionismo prevalece enquanto abordagem pedagógica das propostas de formação continuada, mas devido ao número reduzido de estudos analisados, não podemos responder se este dado pode ser representativo a nível de Brasil. O que nos fica claro diz respeito à influência que as abordagens formativas podem trazer aos professores enquanto aprendizes de TIC. Dado que confirmamos na exposição da tabela $1 \mathrm{em}$ relação ao papel do professor enquanto aprendiz de TIC. Vale considerar neste dado, a importância dos contextos de formação continuada mais concentrados na aprendizagem docente em TIC, do que na técnica como meio de instrução. Nesse sentido, podemos ressaltar que as tecnologias digitais vão além das ferramentas pedagógicas, e que assumem um papel estruturante na forma de pensar, de comunicar e de representar (Almeida; Valente; 2011), acrescentando ainda que este papel estruturante também se encontra na ação, na reflexão e no compromisso docente diante do século XXI. Motivo este de nossa atenção à situacionalidade docente em TIC como um conjunto de fatores que impactuam nas respostas dos professores a este século.

Tabla 1.

As abordagens Pedagógicas e o papel do profesor enquanto aprendiz de TIC

\begin{tabular}{|c|c|c|c|}
\hline Autores & Ano & $\begin{array}{c}\text { Abordagem } \\
\text { Pedagógica/modelo }\end{array}$ & Papel Docente \\
\hline $\begin{array}{l}\text { Lobo da } \\
\text { Costa e } \\
\text { Prado }\end{array}$ & 2013 & $\begin{array}{l}\text { Exploratório- } \\
\text { Investigativa }\end{array}$ & $\begin{array}{l}\text { Construtor } \\
\text { /Reflexivo }\end{array}$ \\
\hline Pupo & 2013 & Construcionismo & $\begin{array}{l}\text { Construtor } \\
\text { /Reflexivo }\end{array}$ \\
\hline Ferreira & 2015 & Construcionismo & $\begin{array}{l}\text { Construtor } \\
\text { /Reflexivo }\end{array}$ \\
\hline $\begin{array}{l}\text { Alcântara, } \\
\text { Dullius e } \\
\text { Carreira }\end{array}$ & 2015 & $\begin{array}{l}\text { Mentorin (Amado, } \\
\text { 2007) }\end{array}$ & Reflexivo \\
\hline Lima & 2015 & Construcionismo & $\begin{array}{l}\text { Construtor } \\
\text { /Reflexivo }\end{array}$ \\
\hline Santos & 2016 & Narrativas Digitais & $\begin{array}{c}\text { Autor do } \\
\text { conhecimento }\end{array}$ \\
\hline Lang & 2016 & Construtivismo & $\begin{array}{l}\text { Construtor } \\
\text { /Reflexivo }\end{array}$ \\
\hline $\begin{array}{l}\text { Prado e } \\
\text { Lobo Costa }\end{array}$ & 2016 & Construcionismo & $\begin{array}{l}\text { Construtor } \\
\text { /Reflexivo }\end{array}$ \\
\hline
\end{tabular}

Outra evidência importante reporta as influências das abordagens formativas no papel do professor enquanto aprendiz de TIC. De acordo com os estudos, que não mencionaram a ideia do papel do professor enquanto aprendiz, mas que relataram os diagnósticos e avanços dos professores ao longo do contexto de formação, as abordagens pedagógicas tem relação com a aprendizagem docente, e se manifestam nos professores por meio da relação dos mesmos com a proposta formativa (conteúdo) e sua conexão tanto com o conhecimento pedagógico quanto com o conhecimento tecnológico (TPACK). Deste modo, a reação dos professores em relação ao modus operandi do contexto de formação pode demonstrar um reflexo de sua práxis enquanto docente. Numa abordagem tradicional, por exemplo, a influência seria a acomodação, à técnica e a reprodução da mesma em sala em sala de aula. Mas no caso dos estudos abarcados em abordagens mais dinâmicas, encontramos o professor mais envolvido no proceso formativo, que se motiva, que passa a refletir as situações de sala de aula em conjunto com o seu grupo de estudo. Passa a relatar suas dificuldades e a pensar sobre os condicionantes das mesmas. Passa, nesse sentido, a ter consciência crítica da sua ação, da sua reflexão e do seu compromisso em TIC.

Interessante colocar que os estudos analisados trabalhavam o contexto da formação continuada como uma espécie de problematização do uso das TIC em sala de aula, partindo da abordagem pedagógica o sentido apontado. $\mathrm{Na}$ abordagem construcionismo, como a que mais foi utilizada pelos estudos, pudemos perceber a conexão da mesma em relação à ideia de aprendizagem docente amparada pelo fator reconstrução da prática ( Pupo, 2013; Ferreira, 2015; Lima, 2015; Prado \& Lobo da Costa, 2016). Neste achado também visualizamos a crescente atenção à integração dos conhecimentos do conteúdo, da pedagogia, e da tecnología, confirmando mais uma vez o TPACK como elemento de intervenção estruturante dos procesos de formação continuada.

Declarada como uma teoria de fundamentação à ação investigativa de Alcântara, Dullius e Carreira (2015) encontramos de modo particular a ideia de mentoring concebida como:

"uma relação que se estabelece entre duas pessoas, semelhante à do mestre e do discípulo, na filosofia grega, mas também análoga à que pode acontecer entre um mestre e um aprendiz de uma profissão" (Amado, 2007, p. 170).

No que se refere à formação, a ideia do mentorin foi explorada de forma a relacionar mentor e aprendiz, ambos inseridos num propósito planejado para o alcance de objetivos predefinidos (Amado, 2007). Com base neste quadro Alcântara, Dullius e Carreira (2015) utilizaram o modelo com profesores que estavam iniciando seu contato com as tecnologias em sala de aula, em específico com uma professora de Educação Básica, buscando conhecer os detalhes da trajetória de formação da mesma.

Em Santos (2016) encontramos a ideia de posicionar as Narrativas Digitais (ND) como abordagem pedagógica, visando facilitar o proceso de letramento digital e aprendizagem docente. O estudo de Lang (2016) aponta atenção especial à abordagem construtivista, envolvendo-a em sua proposta de formação continuada de forma reflexiva junto aos participantes da mesma. $\mathrm{O}$ referido autor apostou em leituras e no diálogo reflexivo dos participantes da formação em relação à referida abordagem, tendo em vista a problematização dos contextos de sala de aula.

Já Lobo da Costa e Prado (2016) trazem um estudo referente a uma formação que teve como base atividades exploratórios-investigativas, colocando que as mesmas "são tarefas abertas para que os aprendizes gerem 
hipóteses, testem suas conjecturas e procurem por diferentes soluções" (Lobo da Costa \& Prado, 2016, p. 5147).

Vale ressaltar que mesmo os estudos que contemplaram a formação continuada sem referência a expressão específica "abordagem pedagógica", demonstraram uma preocupação implícita com a aprendizagem docente e com a problematização das TIC dentro do contexto de sala de aula. A ideia do professor enquanto aprendiz de TIC esteve presente na referida preocupação, embora não assumindo esta conotação. No entanto, destaca-se também que a ideia de trabalho em grupo, de cooperação e de colaboração entre os pares das formações propostas não se apresentou como referência pontual, mas que vai se redescobrindo como necessária no desafio de integração das TIC aos saberes docentes.

Mais um fator importante a considerar na análise que fizemos dos estudos incluídos tem relação com o conceito de situacionalidade docente em TIC. Foi notável que os trabalhos de formação, em grande maioria, consideraram um diagnóstico como ponto de partida para o desenvolvimento de um contexto de formativo. Esta iniciativa, além de cientificamente correta, vem de encontro com nossa consideração em relação a realidade docente em TIC, e por tanto, em relação às necessidades e também avanços do professor. Visualizamos que a atenção à situacionalidade docente em TIC caminha na direção da democratização das tecnologias digitais aos saberes docentes. Ela aponta para o professor como um sujeito de direitos, sem julgamentos ou interpretações ingénuas de seu papel, concebendo-o dentro do cenário onde humanismo e tecnologia não se excluem (Freire, 1979).

\section{Discussão}

O resultado da análise dos estudos vem revelar que muitas são as iniciativas que se apresentam em relação aos processos de formação continuada de professores. Outro argumento que trazemos é que existe uma crescente atenção às abordagens pedagógica em relação à aprendizagem docente, movimento este capaz de preencher a lacuna existente entre o formar para a técnica, e o formar para a conscientização crítica em relação aos potênciais das TIC. Neste ponto nos baseamos em Freire (1979) que traz a questão do ímpeto criador do homem. Para ele a criação faz parte de todo homem, reforçando que a educação se torna mais autêntica a partir do desenvolvimento deste ímpeto criador. Todavia, é preciso considerar que "a educação deve ser desinibidora e não restritiva" (Freire, 1979, p. 17), e isso vale para a questão dos processos de formação docente.

Neste raciocínio encontramos o reforço da necessidade da consciência crítica do profesor perante a sua relação com as Tecnologias Digitais, sendo fato que a medida que discute a sua realidade em TIC, inicia em conjunto, um processo de busca da superação de suas dificuldades. Neste exercício, o profesor passa a oferecer respostas às demandas do Século XXI de forma a temporalizar os espaços, fazendo "história pela sua própria atividade criadora" (Freire, 1979, p. 17) em TIC.
Por esta via, e como exemplo, podemos citar Valente (1998) e sua consideração à abordagem construcionista, relacionando-a ao professor que participa de um processo de capacitação em TIC. Nesta abordagem por exemplo, o professor se constitui enquanto um sujeito construtor do conhecimento, assumindo um papel mais dinâmico e ativo dentro dos contextos de capacitação. Neste sentido, a interação docente com a tecnologia dentro deste contexto vem significar o meio para possibilitar a construção do conhecimento pelo professor, e este deixa de fazer parte dos processos formativos que "transferem" conhecimentos a respeito de tecnologia sob a égide da instrução. Ainda em Valente (1989, p. 4) podemos considerar que a abordagem construcionista dá ênfase a aprendizagem, e é por este ponto que ressaltamos a importância do processo de ensino e aprendizagem dos professores em TIC, reconhecendo que as abordagens de formação exercem influência no papel docente enquanto aprendiz e na qualidade desta aprendizagem em TIC. Este ponto é reconhecido por nós como um dos fatores da situacionalidade docente em TIC, porque o professor enquanto espectador de contextos de formação (Koehler \& Mishra, 2005) não consegue construir conhecimentos, e esta questão tem impacto na ação, na reflexão e no compromisso dos professores diante das TIC. Neste cenário, podemos situar o professor no papel de espectador dentro da abordagem instrucionista, ou seja, aquele que recebe o ensino, que memoriza e depois reproduz a técnica. Todavia, esta abordagem pode levar o professor a conceber o uso das TIC em sala de aula como algo complicado e que requer um manual de instrução.

$\mathrm{Na}$ contramão deste pensamento enquadramos as abordagens que visam o aprendizado docente de forma concreta, considerando o que evidenciamos nas propostas de formação que problematizaram o uso das TIC em contexto de sala de aula. É neste sentido que enquadramos o professor enquanto aprendiz de TIC, num movimento de formação que o leve a pensar, agir e refletir junto com as potencialidades tecnológicas. $\mathrm{O}$ seu papel, assim, enquanto aprendiz de TIC, se define na postura dinâmica de um construtor do conhecimento ou, de acordo com Koehler e Mishra (2005), no papel de designer, problematizando os desafíos de sala de aula de forma a integrar conteúdo, pedagogia e tecnologia dentro do contexto formativo.

\section{Considerações finais}

O elencado por este artigo de RSL retrata o desafio de integração dos professores ao cenário das Tecnologia Digitais. Ele reporta a realidade dos professores carentes de formação, e também apresenta os esforços alavancados por meio de investigações científicas para compreender e dar respostas à esta realidade. Nesse sentido, abarca tanto a experiência dos professores em sua prática escolar quanto a intencionalidade das propostas de formação, prevendo contudo, apontar as evidências que atentam para as possibilidades diferenciadas de interação docente com as tecnologias. No entanto, considerando que

"Não há transição que não implique um ponto de partida, um processo e um ponto de chegada. Todo o amanhã se cria 
num ontem, através de um hoje. De modo que o nosso futuro baseia-se no passado e se corporifica no presente. Temos que saber o que fomos e o que somos, para sabermos o que seremos". (Freire, 1979, p. 18)

Nesse sentido, temos que é preciso apostar na reflexão crítica das abordagens pedagógicas pertinentes às tecnologias digitais, ao mesmo tempo em que se confere a necessária atenção aos processos de ensino e de aprendizagem dos professores, haja visto que constituem espaços para o diálogo entre as demandas sobre tecnologia e docência e a situacionalidade docente em TIC.

Embora parecemos insistentes na exposição da expressão situacionalidade docente em TIC neste artigo, o fazemos porque damos credibilidade ao potencial também existente no professor enquanto um constante aprendiz, não só em relação a seu ofício, mas sobretudo, em relação ao desenvolvimento de humanidades.

\section{Referencias}

Abrúcio, L. F. (Org.). (2016). Formação de professores no Brasil : diagnóstico, agenda de políticas e estratégias para a mudança. São Paulo: Moderna.

Alcântara, L. A. G.; Dullius, M. M; Carreira, S. P. G. (2016). O desenvolvimento do professor: uma proposta de formação continuada centrada nas tecnologias e ancorada na prática. REMAT: Revista Eletrônica da Matemática, v. 1, n. 2. Disponível em:<https://periodicos.ifrs.edu.br/index.php/REMAT/ article/view/1223>. Acesso em: 21 mar. 2017.

Almeida, M. E. B., \& Valente, J. A. (2011). Tecnologias e currículo: trajetórias convergentes ou divergentes? São Paulo: Paulus.

Amado, N. M. P. (2007). O professor estagiário de Matemática e a integração das tecnologias na sala de aula: relações de mentoring numa constelação de práticas. Tese (Doutoramento em Matemática Especialidade em Didática da Matemática). Universidade do Algarve.

Boland A, Cherry G, Dickson R. (2013). Doing a Systematic Review. SAGE Publications Ltd.

Lobo da Costa, N. M. L.; Prado, M. E. B. B. (2013). Formação continuada e uma abordagem exploratórioinvestigativa em geometria espacial de posição. Actas del VII CIBEM ISSN, v. 2301, n. 0797, p. 5143. Disponível em: http://cibem7.semur.edu.uy/7/actas/pdfs/212.pdf>. Acesso em 22 de mar. 2017.

Critical Appraisal Skills Programme. (2017). CASP Qualitative Checklist. Oxford. Disponível em < http://www.casp-uk.net/casp-tools-checklists>. Acesso em 10 de abr. 2017.

Ferreira, G. K. F. (2015). Formação continuada de professores para o uso das tecnologias educativas: um estudo de caso sobre o discurso e a prática. 416f. Tese (Doutorado). Universidade do Minho, Instituto de Educação, Portugal.

Freire, P. (1979). Educação e mudança. 12ª Ed. Editora Paz e Terra: Rio de Janeiro.
Koehler, M. J., \& Mishra, P. (2005). Teachers learning technology by design. Journal of computing in teacher education, 21(3), 94-102.

Lang, A. M. R. (2016). $O$ desenvolvimento do conhecimento pedagógico tecnológico do conteúdo de professores do ensino fundamental. $123 \mathrm{f}$. Dissertação (Mestrado) - Instituto de Biociências da Universidade Estadual Paulista "Júlio de Mesquita Filho" - Campus de Rio Claro.

Lima, S. C. (2015). Parcerias digitais e a formação do professor de língua portuguesa: um estudo à luz da teoria da atividade. $230 \mathrm{f}$. Programa Interdisciplinar de Pós-Graduação em Linguística Aplicada da Universidade Federal do Rio de Janeiro, Rio de Janeiro, 2015.

Maneira, S.; Martins, A.; Gomes, M. J. (2016). Technological Pedagogical Content Knowledge and continuous training of in-service teachers: a Systematic Review of the Literature, Sevilla, ICERI2016 Proceedings, Espanha, pp. 1040-1048.

Prado, M. E. B. B.; Lobo da Costa, N. M. (2016). O papel da atividade de programação no processo de construção de conhecimentos para a docência. Revista $e$ Curriculum, v. 14, n. 3. Disponível em: http://www.redalyc.org/html/766/76647706007/.

Acesso em: 22 de mar. 2017.

Que Conceito. (2015). Homepage. Disponível em: < http://queconceito.com.br/>. Acesso em: 15 de abr. 2017.

Pupo, R. A. (2013). O uso das tecnologias digitais na formação continuada do professor de matemática. 105f. Dissertação (Mestrado). Programa de PósGraduação da Universidade Bandeirante Anhanguera, São Paulo.

Santos, G. S. V.; et al. (2016). Narrativas digitais na formação de educadores: possibilidades de aturia com o podcast. Monografia (Especialização). Universidade Federal de Santa Catarina, 2016. Disponível em < https://repositorio.ufsc.br/handle/123456789/168839>. Acesso em: 23 mar. 2017.

Valente, J. A. A. (1998). Telepresença na formação de professores na área de informática em educação: implantando o construcionismo contextualizado. In: Congresso Ibero-Americano de Informática na Educação, 4. Brasília. Anais eletrônicos... Brasília: Congresso Ibero Americano de Informática na Educação, 1998. Disponível em: <http://ism.dei.uc.pt/ribie/docfiles/

txt200342421644232-pdf>. Acesso em: 30 maio de 2009.

\section{Nota}

Concessão de Licença $n^{\circ}$ 13.458.152-2 - SEED Governo do Estado do Paraná - Brasil 